NETWORK MODEL TREES

PAYTON J. JONES

HARVARD UNIVERSITY

PATRICK MAIR

HARVARD UNIVERSITY

Thorsten Simon

UNIVERSITÄT INNSBRUCK

AChim ZeILEIS

UNIVERSITÄT INNSBRUCK

Correspondence should be sent to E-Mail: payton_jones @g.harvard.edu Phone: 617-495-3800

Website: https://scholar.harvard.edu/paytonjones/home 


\title{
NETWORK MODEL TREES
}

\begin{abstract}
:
In many areas of psychology, correlation-based network approaches (i.e., psychometric networks) have become a popular tool. In this paper we define a statistical model for correlation-based networks and propose an approach that recursively splits the sample based on covariates in order to detect significant differences in the network structure. We adapt model-based recursive partitioning and conditional inference tree approaches for finding covariate splits in a recursive manner. This approach is implemented in the networktree $\mathrm{R}$ package. The empirical power of these approaches is studied in several simulation conditions. Examples are given using real-life data from personality and clinical research.
\end{abstract}

Key words: Network Analysis, Correlation Networks, Recursive Partitioning, Decision Trees, Conditional Inference. 


\section{Introduction}

Network science is part of the larger field of complex systems, which studies how interactions of many parts in a system give rise to emergent properties. Within the past decade, the network approach has gained significant ground as an approach for studying mental disorders as systems of interacting symptoms or other psychobiological mechanisms (McNally, 2019; Borsboom, 2017; Jones et al., 2017). The rapid success of network theory can perhaps be attributed to a historically uncomfortable mismatch between diagnostic manuals (e.g., Diagnostic and Statistical Manual of Mental Disorders American Psychiatric Association, 2013) and cognitive-behavioral models. Many diagnostic manuals implicitly follow a common-cause model that depicts mental disorders as categorical disease entities that give rise to emotional and behavioral symptoms (Borsboom, 2017). In contrast, cognitive-behavioral models emphasize the importance of self-reinforcing feedback loops among transdiagnostic behaviors, emotions, and cognitions, such as the pathological role of avoidance behaviors in reinforcing anxiety (Hanley et al., 2003; Salters-Pedneault et al., 2004). Such interactions are often disallowed or disregarded in common-cause models that view mental disorders as underlying diseases (Borsboom, 2017).

Psychological variables are often measured indirectly using psychometric approaches, necessitating the development of statistical techniques to apply network analytic methods in psychology. These methods have been broadly dubbed network psychometrics, and typically involve estimating conditional dependence graphs from covariance matrices (Epskamp et al., 2017, 2018; Marsman et al., 2018). In addition to their role in studying mental disorders, network psychometric approaches have been used to study personality (Costantini et al., 2019), attitudes (Dalege et al., 2016), resilience factors (Fritz et al., 2018), and other psychological systems.

Unfortunately, common network psychometric approaches are limited by assumptions of homogeneity. The systems that constitute mental disorders and other psychological phenomena are argued to be extremely heterogeneous (Fried and Nesse, 2015). This means that models aggregated across persons may blur important distinctions in causal mechanisms that are heterogeneous across individuals or groups of individuals. It is possible that networks may vary across gender, age, personality, and a host of additional variables. There is thus a natural marriage between network models and statistical approaches that allow for explicitly modeling heterogeneity using appropriate covariates.

Some existing approaches to modelling network heterogeneity using covariates include exponential random graph models (ERGMs), changepoint analyses, and moderated network models (MNMs). ERGMs are often used to incorporate node or edge covariates in networks of relational data (Harris, 2014), but to our knowledge have not been used in the context of network psychometrics. Changepoint analyses are an example of incorporating a very 
specific covariate to networks: time. Various approaches to changepoint analysis have been discussed in the network psychometric literature, including kernel-based and hidden Markov change point models (Cabrieto et al., 2018; Park and Sohn, 2019). MNMs allow each pairwise interaction between variables in a network to be moderated by covariates (Haslbeck et al., 2018). MNMs are an approach for exploring covariates in networks in a parameterized manner.

In this article we propose an approach that recursively splits the sample based on covariates in order to detect differences in the network parameters (e.g., correlation coefficients). Such recursive partitioning approaches have a long tradition in the statistical literature and became increasingly popular in psychology in recent years (e.g., Strobl et al., 2009; Merkle and Shaffer, 2011; Brandmaier et al., 2013). One benefit of this approach compared to moderation is that it can automatically detect nonlinearities and interactions in an exploratory setting. Whereas MNMs are particularly useful for exploring moderation of individual pairwise interactions, recursive partitioning facilitates interpretations of how covariates are related to heterogeneity across the entire network.

An important development within this context is model-based recursive partitioning (MOB; Zeileis et al., 2008). It is a semi-parametric approach which aims to finds splits with respect to parameters of a particular underlying model. In other words, we have a model as "response" in each terminal node, rather than a single dependent variable only as in classical tree approaches like CART (classification and regression trees; Breiman et al., 1984). Using the MOB algorithm the splits are determined in a way such that the model parameters are maximally heterogeneous across the terminal nodes. In the psychometric literature the MOB idea has been adapted to several model families: Bradley-Terry models (Strobl et al., 2011), Rasch models (Strobl et al., 2015; Komboz et al., 2018), factor analysis and structural equation models (Merkle and Zeileis, 2013), multinomial processing trees (Wickelmaier and Zeileis, 2018), and generalized linear mixed-effects models (Fokkema et al., 2018).

Another recursive partitioning approach proposed in the statistical literature are conditional inference trees (CTree; Hothorn et al., 2006b). CTree is very similar to MOB in many respects but does not have to be based on a formal parametric model. Instead, CTree is based on a general class of permutation tests which can be combined with parametric scores, but can also be based on completely nonparametric test statistics. While originally introduced for simple decision trees with mean responses in the terminal nodes, CTree has also recently been leveraged to obtain trees with fitted parametric models in each node (see e.g., Seibold et al., 2016).

In this article we integrate both MOB and CTree into network models in order to find optimal subgroup splits which lead to heterogeneous network structures in the terminal nodes. We begin with technical elaborations on MOB and CTree within the context of 
network models, with the formulation of a multivariate normal model at its core. Similarities and differences between the two approaches are discussed. Next, some simulation studies are performed exploring sensitivity and specificity of MOB and CTree networks. This is followed by a real-life application. R ( $\mathrm{R}$ Development Core Team, 2019) code for fully reproducing this example is provided in the supplemental materials, using the networktree package (Jones et al., 2018).

\section{Network Model Tree Theory}

This section formally introduces the network model tree (NMT) approach. We develop the NMT theory for both MOB and CTree. The basic workflow, inherent to both tree approaches, is the following:

1. fit a multivariate normal distribution to the response in the current sub-sample;

2. compute a goodness-of-fit measure for the parameters of the multivariate normal distribution (e.g., based on the log-likelihood);

3. assess whether this measure is linked to any of the partitioning variables;

4. if this is the case, find the split that yields the highest improvement of the goodnessof-fit measure.

These steps are repeated until no further split would increase the goodness-of-fit measure.

Even though MOB and CTree are conceptually similar, there are some differences in how MOB and CTree tackle these four steps. In Step 2, MOB uses parameter instability tests based on the multivariate normal distribution, whereas CTree uses permutation tests of empirical correlations. In Step 4, MOB maximizes a partitioned log-likelihood, whereas CTree maximizes a two-sample statistic. A systematic comparison of these two approaches follows below, after a formal introduction of MOB and CTree networks.

\section{MOB Networks}

Since the network models considered in this paper are based on Pearson correlations, we assume an underlying multivariate normally distributed $p$-dimensional random variable $Y$ with individual observations $\mathbf{y}_{i}(i=1, \ldots, n$; collected as $n \times p$ matrix $\mathbf{Y})$. The corresponding density function with mean vector $\boldsymbol{\mu}$ and variance-covariance matrix $\boldsymbol{\Sigma}$ is

$$
f\left(\mathbf{y}_{i} ; \boldsymbol{\mu}, \boldsymbol{\Sigma}\right)=\frac{1}{\sqrt{(2 \pi)^{p}|\mathbf{\Sigma}|}} \exp \left(-\frac{1}{2}\left(\mathbf{y}_{i}-\boldsymbol{\mu}\right)^{\prime} \boldsymbol{\Sigma}^{-1}\left(\mathbf{y}_{i}-\boldsymbol{\mu}\right)\right) .
$$

Let $\mathbf{D}$ be the diagonal matrix with the reciprocals of the square roots of the diagonal entries in $\boldsymbol{\Sigma}$ on its diagonal. Then $\mathbf{R}=\mathbf{D} \boldsymbol{\Sigma} \mathbf{D}^{\prime}$ is the $p \times p$ correlation matrix. 
Let $\boldsymbol{\theta}=\left(\boldsymbol{\mu}, \boldsymbol{\sigma}^{2}, \boldsymbol{\rho}\right)$ be the full parameter vector of dimension $k$ containing $p$ means in $\boldsymbol{\mu}$, $p$ variances in $\boldsymbol{\sigma}^{2}$, and $p(p-1) / 2$ correlations from the lower triangular part of $\mathbf{R}$, collected in $\boldsymbol{\rho}$. The associated log-likelihood is

$$
\begin{aligned}
\ell\left(\boldsymbol{\theta} ; \mathbf{y}_{1}, \ldots, \mathbf{y}_{n}\right) & =\sum_{i=1}^{n} \ell\left(\boldsymbol{\theta}, \mathbf{y}_{i}\right)=\sum_{i=1}^{n} \log f\left(\mathbf{y}_{i} ; \boldsymbol{\theta}\right) \\
& =\sum_{i=1}^{n}-\frac{1}{2}\left(\left(\mathbf{y}_{i}-\boldsymbol{\mu}\right)^{\prime} \boldsymbol{\Sigma}^{-1}\left(\mathbf{y}_{i}-\boldsymbol{\mu}\right)+\log |\boldsymbol{\Sigma}|+p \log (2 \pi)\right) .
\end{aligned}
$$

Taking the partial derivatives leads to the score function $^{1}$

$$
\mathbf{s}\left(\boldsymbol{\theta} ; \mathbf{y}_{i}\right)=\left(\frac{\partial \ell\left(\boldsymbol{\theta} ; \mathbf{y}_{i}\right)}{\partial \theta_{1}}, \ldots, \frac{\partial \ell\left(\boldsymbol{\theta} ; \mathbf{y}_{i}\right)}{\partial \theta_{k}}\right)^{\prime}
$$

Solving $\sum_{i=1}^{n} \mathbf{s}\left(\hat{\boldsymbol{\theta}} ; \mathbf{y}_{i}\right)=\mathbf{0}$ gives the maximum likelihood $(\mathrm{ML})$ estimates $\hat{\boldsymbol{\theta}}=\left(\hat{\boldsymbol{\mu}}, \hat{\boldsymbol{\sigma}}^{2}, \hat{\boldsymbol{\rho}}\right)$. Note that in the case of a multivariate normal distribution without further covariates no numeric optimization is required since there are closed form solutions for all the parameters in $\boldsymbol{\theta}$.

The score function is of key importance in the MOB approach since it acts as a goodnessof-fit measure (i.e., the contribution of observation $i$ to each of the $k$ parameters). The main idea behind MOB is to find out whether parameters are stable over covariate splits or not. The score functions $\mathbf{s}\left(\boldsymbol{\theta} ; \mathbf{y}_{i}\right)$ are used as (in)stability measures, assessing whether there is a systematic deviation from 0 over a partitioning variable $Z$, or they just fluctuate randomly around 0 .

To explicitly test for significant covariate splits, we adopt the invariance testing framework proposed by Merkle and Zeileis (2013). Note that for notation convenience we limit our elaborations to a single partitioning variable $Z$ only. The extension to the practically relevant case of multiple variables $Z_{1}, \ldots, Z_{q}$ is straightforward. The starting point for the invariance testing framework is the cumulative score process

$$
\mathbf{B}(t ; \hat{\boldsymbol{\theta}})=\hat{\mathbf{I}}^{-1 / 2} n^{-1 / 2} \sum_{i=1}^{\lfloor n t\rfloor} \mathbf{s}\left(\hat{\boldsymbol{\theta}} ; \mathbf{y}_{(i)}\right) .
$$

$\hat{\mathbf{I}}$ is an estimate of the covariance matrix of the scores, $\lfloor n t\rfloor$ is the floor function of $n t$, and $\mathbf{y}_{(i)}$ denotes the individual with the $i$-th smallest value with respect to the partitioning variable $Z$. Therefore, (4) defines a scaled cumulative sum of ordered scores. The observed values of $t$ are restricted to the set $\{0,1 / n, 2 / n, \ldots, n / n\}$ such that $\mathbf{B}(t ; \hat{\boldsymbol{\theta}})$ becomes an $n \times k$ matrix with elements $\mathbf{B}(i / n ; \hat{\boldsymbol{\theta}})$. For simplicity in notation we denote this matrix by $\mathbf{B}(\hat{\boldsymbol{\theta}})$,

${ }^{1}$ The derivatives are given in the Appendix. 
with elements $\mathbf{B}(\hat{\boldsymbol{\theta}})_{i j}$. For further details on this process and its asymptotic properties see Hjort and Koning (2002) and Zeileis and Hornik (2007).

Based on $\mathbf{B}(\hat{\boldsymbol{\theta}})$ various test statistics can be constructed, depending on the measurement level of $Z$. We limit our explanations to (maximum) Lagrange multiplier (LM) tests, as presented in Andrews (1993), Merkle and Zeileis (2013), Merkle et al. (2014), and Wang et al. (2014), for which desirable power properties have been shown:

- $Z$ metric $^{2}: \max L M=\max _{i=\underline{i}, \ldots, i}\left\{\frac{i}{n}\left(1-\frac{i}{n}\right)\right\}^{-1} \sum_{j=1}^{k} \mathbf{B}(\hat{\boldsymbol{\theta}})_{i j}^{2}$.

- $Z$ ordinal ( $m$ levels): $\max L M_{o}=\max _{i \in\left\{i_{1}, \ldots, i_{m-1}\right\}}\left\{\frac{i}{n}\left(1-\frac{i}{n}\right)\right\}^{-1} \sum_{j=1}^{k} \mathbf{B}(\hat{\boldsymbol{\theta}})_{i j}^{2}$.

- $Z$ nominal ( $m$ levels): $L M_{u o}=\sum_{l=1}^{m} \sum_{j=1}^{k}\left(\mathbf{B}(\hat{\boldsymbol{\theta}})_{i_{l} j}-\mathbf{B}(\hat{\boldsymbol{\theta}})_{i_{l-1} j}\right)^{2}$.

For $\max L M$, critical values and corresponding $p$-values can be found by approximate asmyptotic techniques (Hansen, 1997), for $\max L M_{o}$ by direct simulation (Zeileis, 2006), and for $L M_{u o}$ through a $\chi^{2}$-distribution, since this statistic is asymptotically equivalent to the usual likelihood-ratio statistic. The variable associated with the smallest significant $p$-value (Bonferroni adjusted) is selected for binary splitting. The split sample in the daughter nodes is then subject to further potential splitting. The algorithm stops when no further significant splits in the partitioning variables can be found. Eventually, the terminal nodes yield a non-overlapping partition of the sample.

An attractive feature of the MOB approach is that only a subset of the parameters in $\boldsymbol{\theta}$ can be subject to invariance testing ("partial structural changes"; Andrews, 1993; Merkle and Zeileis, 2013). For example, in our network approach, we may only be interested in exploring invariance in the correlation parameters $\boldsymbol{\rho}$.

\section{CTree Networks}

In its basic form, CTree finds predictor splits with respect to single or multiple response variables. As opposed to algorithms like CART, CTree uses statistical tests for splitting by embedding the recursive partitioning idea into the permutation test framework proposed by Strasser and Weber (1999, see also Hothorn et al., 2006a). This framework uses independence tests constructed by means of the conditional distribution $D(\cdot)$ of linear hypotheses.

In the CTree approach we operate on standardized versions of the variables in $\mathbf{Y}$, since it is sufficient to derive a proportionality expression for the correlation vector involving a simple vector multiplication, as formalized below. Therefore, let $\mathbf{Y}^{*}$ be the $n \times p$ data matrix $(i=1, \ldots, n)$ of standardized variables, and $\mathbf{Z}$ be the $n \times q$ matrix $(j=1, \ldots, q)$ of

\footnotetext{
${ }^{2}[\underline{i}, \bar{i}]$ denotes the interval for the potential change point shift $(\underline{i}$ as the minimum segment size, $\bar{i}=n-\underline{i})$.
} 
covariates. The $q$ partial hypotheses of independence are $H_{0}^{j}: D\left(\mathbf{Y}^{*} \mid Z_{j}\right)=D\left(\mathbf{Y}^{*}\right)$, with the global hypothesis being $H_{0}: \bigcap_{j=1}^{q} H_{0}^{j}$. To test the partial hypotheses, the following linear test statistics is used:

$$
\mathbf{T}_{j}=\operatorname{vec}\left(\sum_{i=1}^{n} w_{i} g_{i}\left(z_{i j}\right) h\left(\mathbf{y}_{i}^{*},\left(\mathbf{y}_{1}^{*}, \ldots, \mathbf{y}_{n}^{*}\right)\right)^{\prime}\right) .
$$

For the global hypothesis, the test statistic is

$$
\mathbf{T}=\operatorname{vec}\left(\sum_{i=1}^{n} w_{i}\left(g_{1}\left(z_{i 1}\right)^{\prime}, \ldots, g_{m}\left(z_{i m}\right)^{\prime}\right)^{\prime} h\left(\mathbf{y}_{i}^{*},\left(\mathbf{y}_{1}^{*}, \ldots, \mathbf{y}_{n}^{*}\right)\right)^{\prime}\right)
$$

In these equations, $w_{i}$ acts case weight. Each node in the tree is represented by a weight vector $\mathbf{w}=\left(w_{1}, \ldots, w_{n}\right)$. If the corresponding observation $i$ is in the node, $w_{i}=1$; if not, $w_{i}=0$. The covariate transformation function is $g_{i}(\cdot)$. If a covariate $Z_{j}$ is metric, the identity function is used. In case of nominal $Z_{j}$, dummy vectors are computed. For ordinal $Z_{j}$, scores are assigned to ordered factors.

The critical ingredient for adapting these statistics to network applications is the definition of a suitable influence function $h(\cdot)$, which defines the transformation of the responses. We make use of the following proportionality relation of a correlation coefficient $\rho_{k k^{\prime}}$ with respect to two standardized column vectors $\mathbf{y}_{k}^{*}$ and $\mathbf{y}_{k^{\prime}}^{*}\left(k \neq k^{\prime} ; k, k^{\prime}=1, \ldots, p\right)$.

$$
\rho_{k k^{\prime}} \propto\left\langle\mathbf{y}_{k}^{*}, \mathbf{y}_{k^{\prime}}^{*}\right\rangle
$$

The inherent element-wise multiplication of the vector elements $\mathbf{y}_{k}^{*} \circ \mathbf{y}_{k^{\prime}}^{*}$ (Hadamard product) results in a new vector $\mathbf{s}_{k k^{\prime}}$ with $n$ elements. Each element gives the contribution of an individual observation to the correlation $\rho_{k k^{\prime}}$, and is therefore interpretable as score function (cf. Eq. (3) in the MOB approach). Contributions to the mean and variance could also be potentially considered in a tree based on conditional inference (i.e., CTree).

Hence, the test statistics in Eq. (5) and Eq. (6) can be used to test for differences of correlations across subgroups. The distribution of these test statistics has been worked out theoretically on the basis of the permutation principle. Details can be found in Strasser and Weber (1999). If the global $H_{0}$ cannot be rejected at a pre-specified level $\alpha$ (Bonferroni corrected), the recursive splitting stops. Conversely, if $H_{0}$ is rejected, a split is performed on the basis of the smallest $p$-value from the partial hypothesis testing involving $H_{0}^{j}$.

\section{Some Technical and Computational Remarks}

How does MOB differ from CTree within our network context? The overarching difference is that MOB finds a score for a given model (here, a multivariate normal distribution), whereas CTree finds a model for given scores (as defined by the influence function). At a 
more detailed level, by considering the four steps listed at the beginning of this section, we can say the following.

In the MOB approach, all steps are explicitly built on the parametric multivariate normal model, using the corresponding maximum likelihood inference. Thus, the model fit yields the maximum likelihood estimate of all parameters in Step 1. In Step 2 the goodnessof-fit measures are the ML scores which are used for a parameter instability test in Step 3. Step 4 maximizes the partitioned log-likelihood.

In contrast, the approach based on CTree is set up such that the empirical means and covariance matrix are used in Step 1. Note that this is equivalent to the ML estimate in a normal model (up to potential degrees-of-freedom adjustments). Step 2 then simply uses the corresponding empirical products underlying the correlation matrix as the goodness-of-fit measure. In Step 3 an (asymptotic) permutation test based on these products is used. The split is selected by maximizing the two-sample permutation test statistic in Step 4. Note that, by design, CTree does not necessarily find the optimal likelihood but it is robust to other potential changes (like changes in means and variances).

On a computational note, both MOB and CTree networks are implemented in the networktree package (Jones et al., 2018). Through a methods argument the user can choose between MOB or CTree for network partitioning, the former being more efficient in terms of computing time. It is also possible to specify a particular type of network: correlation, partial correlation, or graphical lasso (e.g., Friedman et al., 2008). The networktree package provides the high-level interface for fitting NMTs and implements MLE and score functions of the multivariate normal distribution. This high-level interface builds on the partykit package (Hothorn and Zeileis, 2015) that provides the infrastructre for recursive partitioning using either the MOB or the CTree approach. Internally, networks are always stored as correlation matrices and $\mathrm{MOB} / \mathrm{C}$ Tree computations are performed as described above. However, depending on the type specified, outputs such as plots are adjusted correspondingly. The visualization of the terminal nodes is done with the qgraph package (Epskamp et al., 2012).

\section{Simulations}

We are interested in how the power of NMT for both MOB and CTree might differ depending on

1. the total sample size $n$;

2. the number of variables $p$ included in the network;

3. the magnitude of change of a single or multiple values in $\boldsymbol{\rho}$;

4. whether additional spurious partitioning variables $Z_{2}, \ldots, Z_{q}$ were included. 
We are also interested in the type I error when no changes occur, or when changes occur to variable means $\mu$ but not to correlations $\boldsymbol{\rho}$. Throughout the simulations, we focus on testing for partial structural changes in the correlation parameters $\boldsymbol{\rho}$. To determine estimates for $p$ and $n$ in realistic scenarios, we relied on a review of recent psychometric network analyses by Haslbeck and Fried (2017): values of $p$ ranged from 6 to 69 with a median of 17, and values of $n$ ranged from 63 to 9282 with a median of 420 . Based on these values, we chose to simulate at values of $n=100,500,1500$, and $p=5,15,25$.

NMTs estimate a $p \times p$ correlation matrix $\mathbf{R}$ containing $p(p-1) / 2$ unique parameters $\boldsymbol{\rho}$ for each partition. To estimate $\boldsymbol{\rho}$, the sample size for each partition $\left(n^{*}\right)$ must be at least as large as the number of values in $\boldsymbol{\rho}$ (i.e., $n^{*} \geq p(p-1) / 2$ per partition after splitting). Therefore, the minimum $n^{*}$ is quadratically larger than $p$, and can be very large at large values of $p$. For example, when $p=5$, a total $n$ of only 20 is potentially sufficient (i.e., $n^{*}=10$ for each partition), but when $p=25$, a total $n$ of 600 or more is required. We only tested situations when $n$ was sufficiently large compared to $p$ to detect at least one split. Thus, plots of simulations shown later are presented in a triangular fashion, with situations with $n^{*}<p(p-1) / 2$ omitted.

We used the GeneNet $\mathrm{R}$ package (Schaefer et al., 2015) to simulate a correlation matrix with a specified $n$ and $p$. The correlation matrix was created by first generating a random matrix of partial correlations with a specified density of 0.5 , and then taking the MoorePenrose generalized inverse of the partial correlation matrix. The full algorithm for matrix generation is outlined in Schaefer and Strimmer (2004). We then simulated two halves of a dataset using this correlation matrix. In the first half, we used the unaltered correlation matrix and specified that all variable means equal zero. In the second half we modified variable correlations $(\Delta \boldsymbol{\rho})$ or variable means $(\boldsymbol{\Delta} \boldsymbol{\mu}) .{ }^{3}$ We then created a partitioning variable $Z_{1}$. This variable was drawn from a normal distribution and was ordered such that the median occurred at the split point. Subsequently, we ran either MOB or CTree on the simulated dataset to see if a split would be detected based on the $Z_{1}$ variable.

\section{Empirical Power of NMT for a Single Change in $\boldsymbol{\rho}$}

For our first simulation, we altered a single correlation value in $\rho$ by $0.1,0.3$, or 0.5 , representing a small, medium, or large correlation according to common effect size conventions. We simulated 50 data sets for each possible combination of $n, p$, and $\Delta \boldsymbol{\rho}$, which gave us an acceptable trade-off between precision and computing time. We then tested whether MOB/CTree detected a split in the dataset in each case. The results are shown in Figure 1.

\footnotetext{
${ }^{3}$ Occasionally, altering $\boldsymbol{\rho}$ caused $\mathbf{R}$ to be non-positive-definite, in which case we located an approximate positive definite solution or discarded the case if an approximate case could not be found.
} 


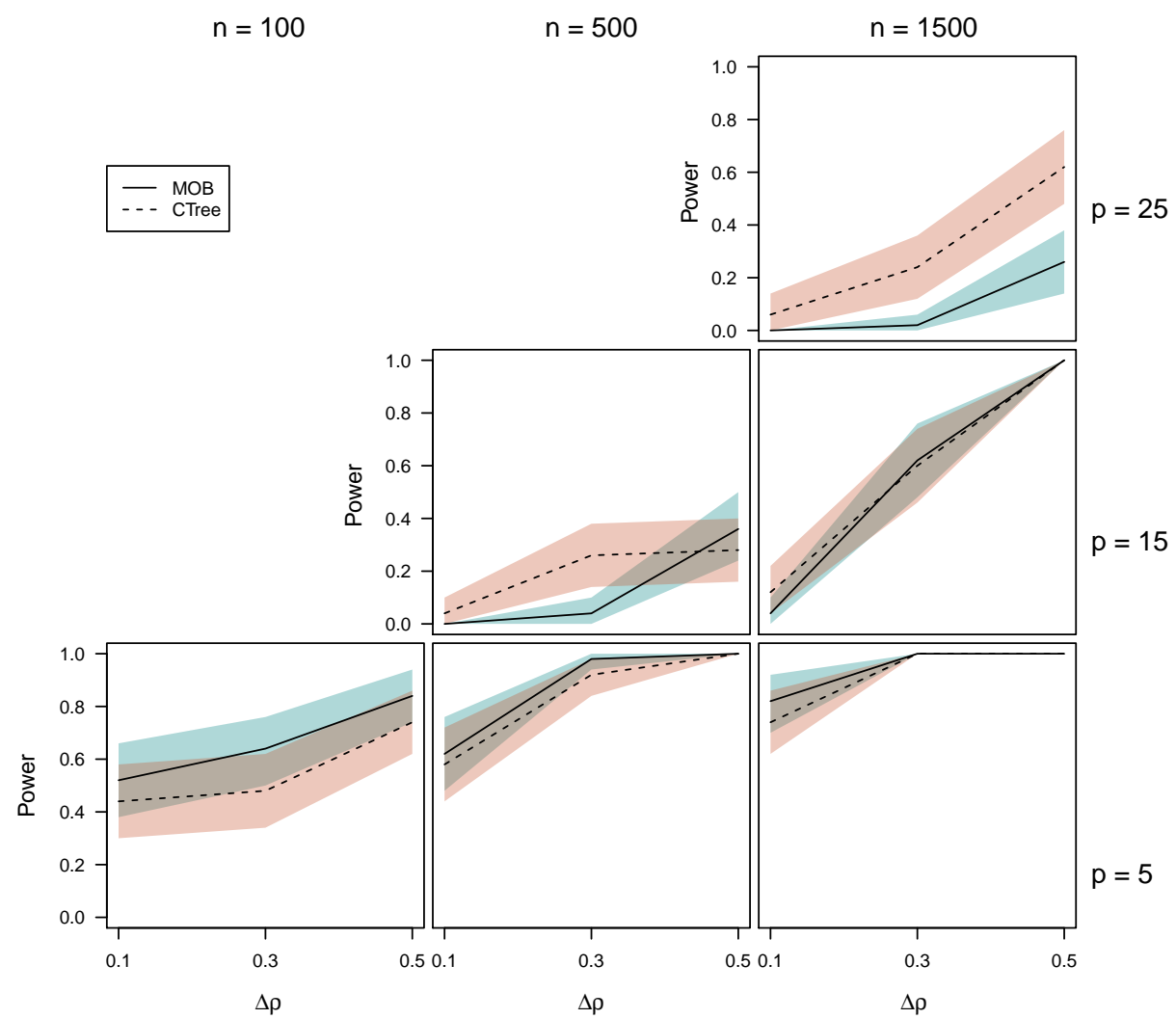

FIGURE 1.

Empirical power for a single change in $\boldsymbol{\rho}$. For each of the six displayed subplots, a single value in $\boldsymbol{\rho}$ was modified as indicated on the $x$ axis. The empirical power is indicated on the $y$ axis with a solid line for MOB and a dashed line for CTree, with shaded $95 \%$ confidence intervals. Each subplot corresponds to specified values of the sample size $n$ and number of nodes $p$, which vary on the $x$ and $y$ axes of the main plot, respectively.

The $x$-axis in each plot represents the degree of alteration in a single value in $\boldsymbol{\rho}$. The $y$-axis represents the empirical power of $\mathrm{MOB} / \mathrm{CTree}$ at each point, calculated as the proportion of simulated datasets in which a split was found. The performance of MOB is indicated by a solid line and the performance of CTree by a dashed line.

The algorithms were able to consistently detect medium-to-large correlation differences in most scenarios, but performance declined significantly at $n=500 / p=15$ and $n=$ $1500 / p=25$. CTree seemed to have a slight advantage in these scenarios. Unsurprisingly, the algorithms did best at a relatively large $n$ and small $p$. 


\section{Empirical Power of NMT for Multiple Changes in $\boldsymbol{\rho}$}

We again altered values in $\boldsymbol{\rho}$ by $0.1,0.3$, or 0.5 , but instead of altering a single value, we altered all values in $\boldsymbol{\rho}$. The results are shown in Figure 2.

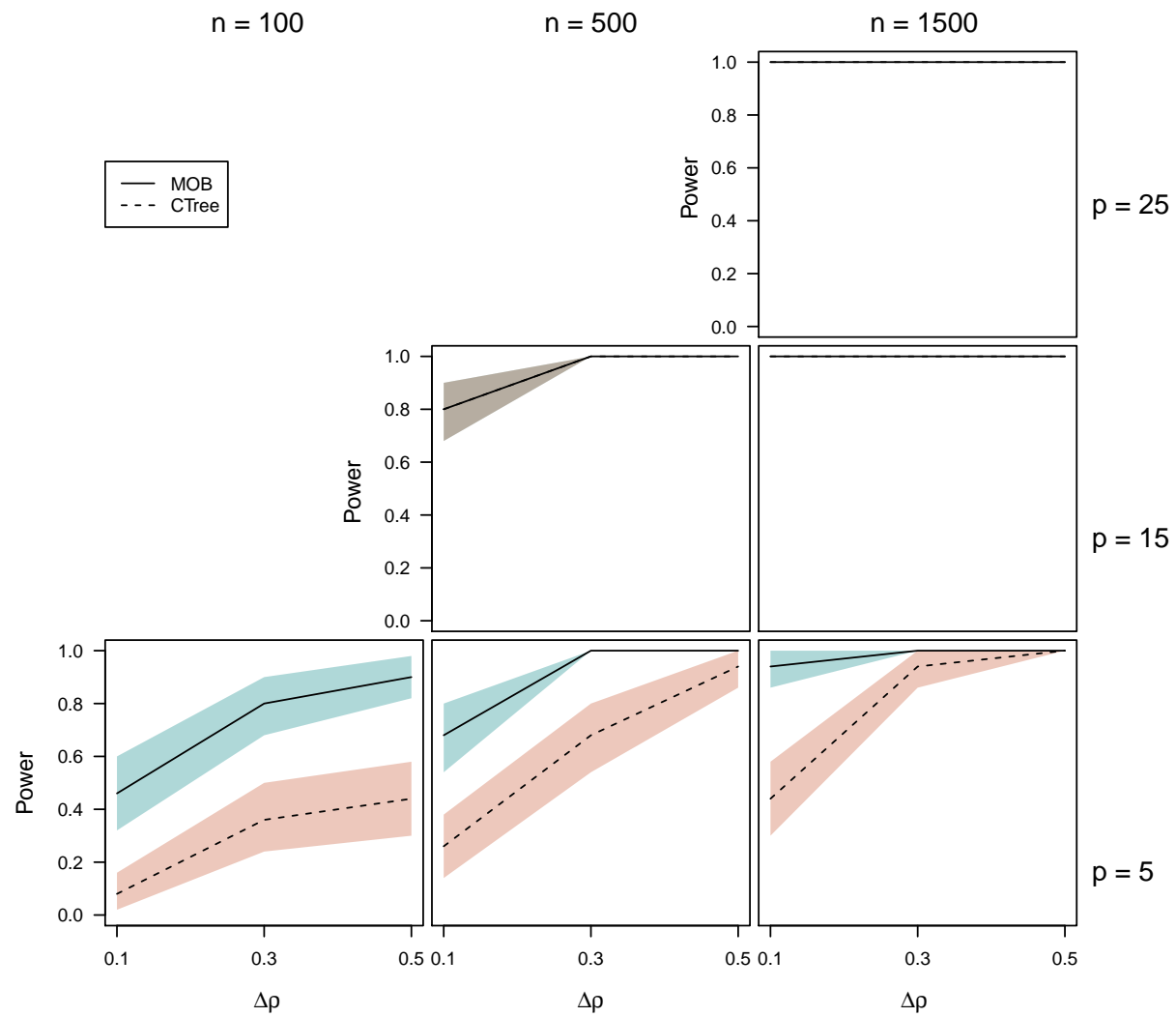

FigURE 2.

Empirical power when changing all values in $\rho$ For each of the six displayed subplots, all values in $\boldsymbol{\rho}$ were modified as indicated on the $x$ axis. The empirical power is indicated on the $y$ axis with a solid line for MOB and a dashed line for CTree, with shaded $95 \%$ confidence intervals. Each subplot corresponds to specified values of the sample size $n$ and number of nodes $p$, which vary on the $x$ and $y$ axes of the main plot, respectively.

Performance drastically improved in nearly all conditions, especially for large values of $p$. This indicates that the algorithms are sensitive to the overall sum of change rather than the proportion of change in $\boldsymbol{\rho}$. At small values of $p$, MOB appeared to show an advantage compared to CTree. 


\section{Empirical Power of NMT when Including Spurious Partitioning Variables}

We were interested in whether including spurious partitioning variables that were unrelated to $\boldsymbol{\rho}$ would reduce the power of MOB/CTree. To test this, we repeated the procedure from the first simulation (changing only 1 value in $\boldsymbol{\rho}$ ) and included 5 spurious partitioning variables $Z_{2}, \ldots, Z_{6}$ in addition to the true partitioning variable $Z_{1}$.

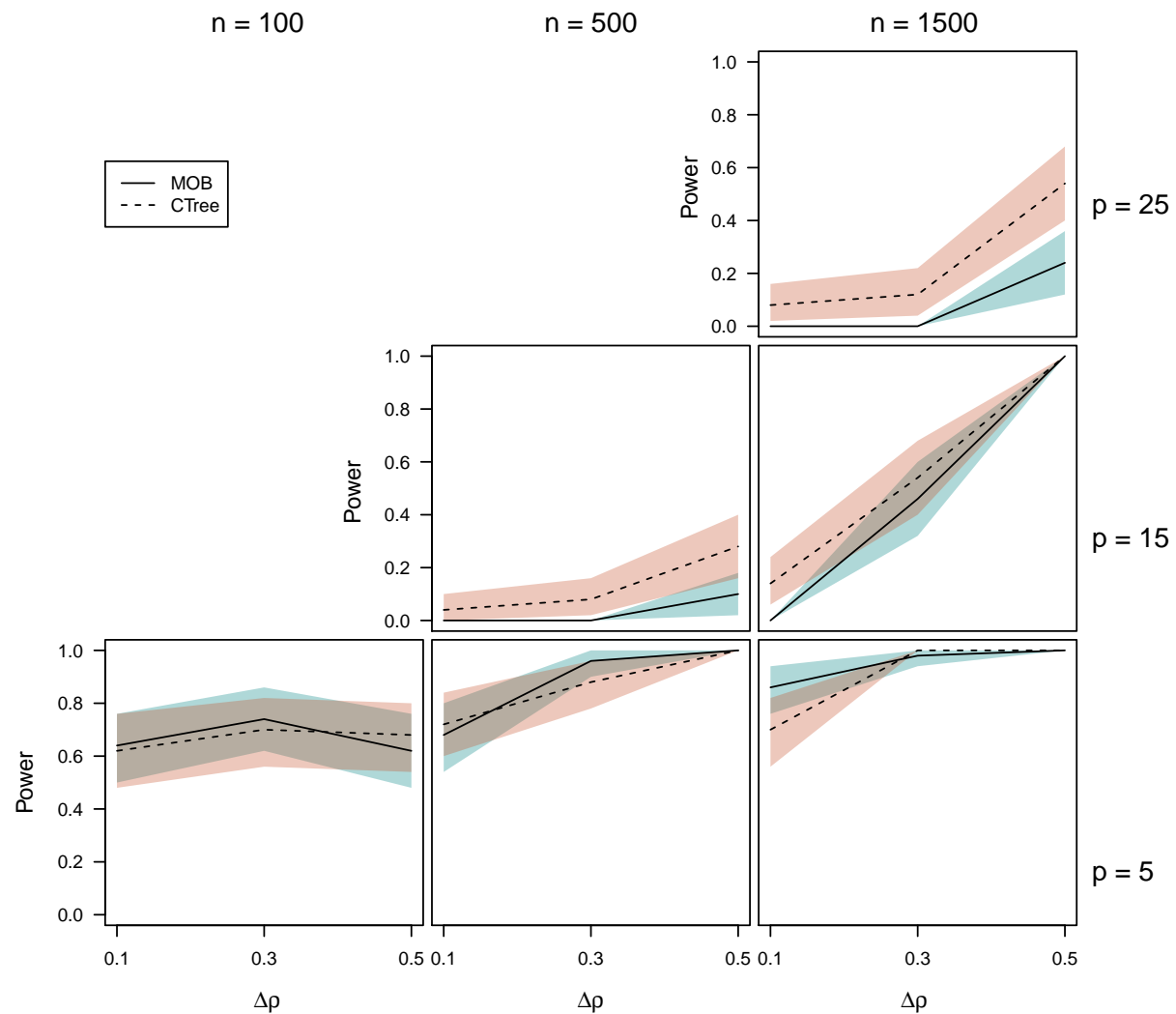

Figure 3.

Empirical power when including spurious partitioning variables. For each of the six displayed subplots, a single value in $\boldsymbol{\rho}$ was modified as indicated on the $x$ axis. In addition, five spurious partitioning variables were added to the model. The empirical power is indicated on the $y$ axis with a solid line for MOB and a dashed line for CTree, with shaded $95 \%$ confidence intervals. Each subplot corresponds to specified values of the sample size $n$ and number of nodes $p$, which vary on the $x$ and $y$ axes of the main plot, respectively.

Results in Figure 3 appeared nearly identical to the first simulation, indicating that including 5 spurious partitioning variables did not meaningfully decrease power. Performance 
appeared to decrease very slightly at $n=500 / p=15$ and $n=100 / p=5$, although this could be a result of small fluctuations due to randomness.

\section{False Positives: Type I Error}

Next, we sought to determine whether the algorithms would detect spurious splits when no true splits existed. Thus, we followed the same simulation paradigm as before, but with $\Delta \boldsymbol{\rho}=0$. We used the same simulated conditions and number of tests per cell as in previous simulations.

TABLE 1.

Type I Error Rate of MOB/CTree

\begin{tabular}{lrrrrrr}
\hline & MOB & \multicolumn{3}{c}{ CTree } \\
\hline & $n=100$ & $n=500$ & $n=1500$ & $n=100$ & $n=500$ & $n=1500$ \\
\hline$k=25$ & & & 0.00 & & & 0.02 \\
$k=15$ & & 0.02 & 0.04 & & 0.02 & 0.04 \\
$k=5$ & 0.02 & 0.02 & 0.02 & 0.04 & 0.02 & 0.08 \\
\hline
\end{tabular}

The results are presented in Table 1 . Overall, we found that the type I error rate was acceptably low for MOB, and acceptably low for CTree in most cases. The type I error rate was 0.08 when $k=5 / n=1500$. It is unclear why the type I error rate was inflated in this cell, and may be due to randomness; the slow runtime of CTree constrained the number of overall tests.

\section{False Positives: Change in Mean}

Relatedly, we were interested if MOB and CTree would detect changes in the correlations when variable means changed, but the correlations remained constant (i.e., $\Delta \rho=0, \Delta \mu \neq$ 0 ). We repeated the same simulation paradigm as in the above simulations but changed the mean of a single variable by a large amount. The results are presented in Table 2 . The results were similar to above $(\Delta \boldsymbol{\mu}=0)$, indicating that changing the mean of a variable does not importantly influence identified splits when testing only for differences in $\Delta \boldsymbol{\rho}$. Minor observed differences between this simulation and the sensitivity simulation are likely due to randomness in a relatively small number of overall simulations, which was constrained by the long runtime of the algorithms.

\section{Application}

In this section, we provide two applications of NMT to empirical data using the networktree package. We used data from the Open Source Psychometrics Project ${ }^{4}$, an organization

\footnotetext{
${ }^{4}$ See https://openpsychometrics.org/.
} 
TABLE 2 .

Detection of a correlation change in MOB (left) and CTree (right) when only the mean was changed

\begin{tabular}{rrrrrrr}
\hline & MOB & \multicolumn{3}{c}{ CTree } \\
\hline & $n=100$ & $n=500$ & $n=1500$ & $n=100$ & $n=500$ & $n=1500$ \\
\hline$k=25$ & & & 0.00 & & & 0.00 \\
$k=15$ & & 0.02 & 0.02 & & 0.00 & 0.04 \\
$k=5$ & 0.00 & 0.02 & 0.00 & 0.02 & 0.00 & 0.00 \\
\hline
\end{tabular}

that maintains an open website for the public to take psychometric tests for educational and entertainment purposes. All de-identified results are maintained in an open database. For the purposes of this section, we show results from MOB (the package default), indicating any differences in CTree in the text.

We selected data from the Ten Item Personality Inventory (TIPI; Gosling et al., 2003), Depression Anxiety and Stress Scale (DASS; Brown et al., 1997), and accompanying demographic information and validity checks.

First, we examined the TIPI data. The TIPI is a brief inventory of the Big Five personality domains. Each personality domain is assessed with two items. One item measures the domain normally and the other item measures the domain in reverse (e.g., "reserved, quiet" for reverse extraversion). After removing individuals that failed validity checks, we were left with 1899 observations. Before applying MOB/CTree, we generated an overall graphical lasso network based on the full sample, given in Figure 4. The structure of this network is characterized by strong negative relationships between the normal and reverse measurements of each domain (e.g., negative relationship between extraversion and extraversion reversed), with complex relationships between separate domains.

Once we had a sense of the network's structure, we moved into partitioning approaches. We used three potential partitioning variables: "engnat" (Is Engligh your native language? Yes/No), "gender" (What is your gender? Male/Female/Other), and "education" (How much education have you completed? Less than high school/High school/University Degree/Graduate Degree [Ordinal]).

The results are shown in Figure 5. The first split occurs between native and non-native English speakers, perhaps indicating a difference in how the items were being interpreted, or perhaps acting as a proxy for unmeasured cultural variables. Among native English speakers, a split is found between those with a high school degree or less, and those with a university or graduate degree. Finally, among those with a university or graduate degree, self-identified females displayed a distinct network from those identifying as male or other. The sample sizes of the terminal nodes ranged from 213 to 847 . The results included some interesting differences in the partitioned networks. For example, native English speakers without a 


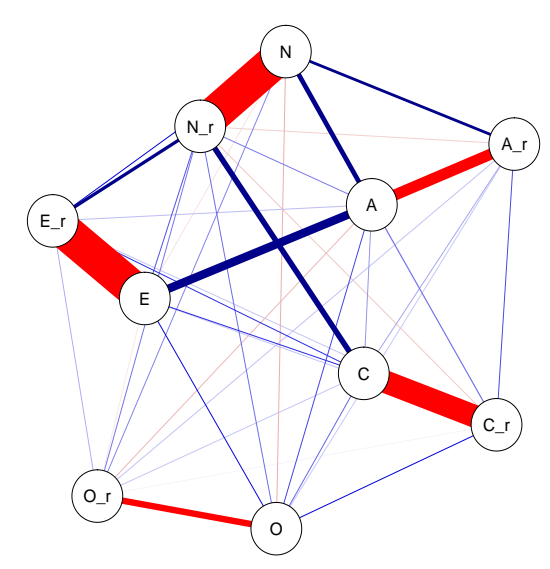

FIGURE 4.

TIPI network from the Open Source Psychometrics Project. Edge thicknesses are determined by the strength of regularized partial correlations between nodes. Blue edges represent positive associations and red edges represent negative associations. Node labels correspond to the first letter of each Big Five personality domain (Extraversion, Neuroticism, Conscientiousness, Agreeableness, and Openness to experience), with the character " $\mathrm{r}$ " indicating items that measure the domain in reverse.

university degree showed a negative relationship between agreeableness and agreeablenessreversed that was significantly weakened in non-native speakers and in native speakers with a university degree. Among native English speakers with a university degree, males and other genders showed a stronger relationship between conscientiousness and neuroticismreversed compared to females. When tested with CTree, a split was found between native and non-native English speakers, but further splits were not identified.

Next, we examined data from the Depression Anxiety and Stress Scale (DASS), a selfreport instrument for measuring depression, anxiety, and tension or stress. We focused specifically on the depression subscale of the DASS. We analyzed a random subset of 5000 individuals from the DASS dataset to maintain a more representative scenario. A network of the complete subset is shown in Figure 6. The depression subscale items were: (1) anhedonia (I couldn't seem to experience any positive feelings at all), (2) getgoing (I just couldn't seem to get going), (3) lookforward (I felt that I had nothing to look forward to), (4) sad (I felt sad and depressed), (5) lostinterest (I felt that I had lost interest in just about everything), (6) worthmuch (I felt I wasn't worth much as a person), (7) worthlife (I felt that life wasn't worthwhile), (8) noenjoy (I couldn't seem to get any enjoyment out of the things I did), (9) downhearted (I felt down-hearted and blue), (10) unenthused (I was unable to become enthusiastic about anything), (11) worthless (I felt I was pretty worthless), (12) nohope (I 


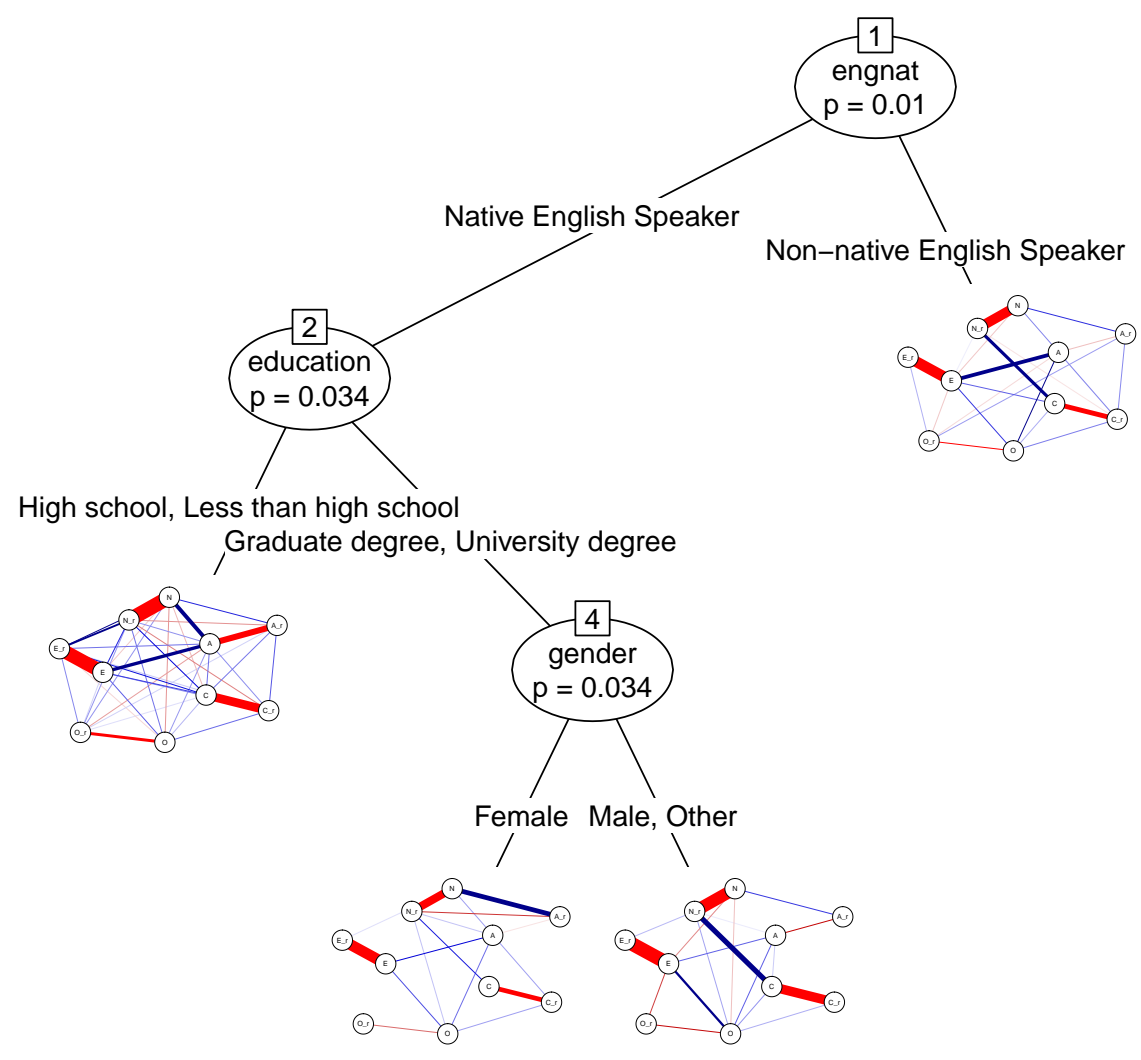

FIGURE 5 .

Partitioning TIPI networks. Partitioning was done using MOB. Edge thicknesses are determined by the strength of regularized partial correlations between nodes. Node labels correspond to the first letter of each Big Five personality domain (Extraversion, Neuroticism, Conscientiousness, Agreeableness, and Openness to experience), with the character " $\mathrm{r}$ " indicating items that measure the domain in reverse.

could see nothing in the future to be hopeful about), (13) meaningless (I felt that life was meaningless), and (14) initiative I found it difficult to work up the initiative to do things.

For the DASS, we used a larger variety of partitioning variables to represent a highly exploratory scenario: "engnat" (Is Engligh your native language? Yes/No), "gender" (What is your gender? Male/Female/Other), "married" (What is your marital status? Never married/Currently married/Previously married), "orientation" (What is your sexual orientation? Heterosexual/Bisexual/Homosexual/Asexual/Other), and "race" (What is your race? Asian/Arab/Black/Indigenous Australian/Native American/White/Other). 


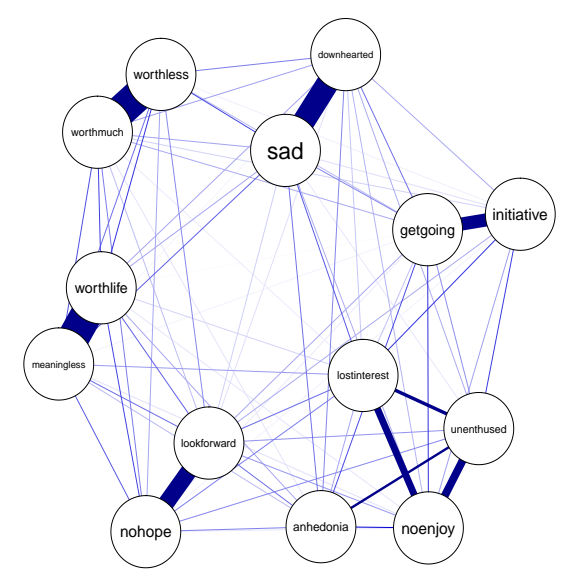

FiguRE 6.

DASS network from the Open Source Psychometrics Project. Edge thicknesses are determined by the strength of regularized partial correlations between nodes. Node labels correspond to the depression subscale items of the DASS.

The results from the DASS are displayed in Figure 7. Again, the primary split occurred between native and non-native English speakers. Among native English speakers, two further splits were found with the race variable. Among the non-native English speakers, a split was found by gender. These results indicate various sources of potential heterogeneity in network structure. Various edge differences differentiated the terminal nodes. For example, among non-native speakers, the connection between worthlife (I felt that life wasn't worthwhile) and nohope (I could see nothing in the future to be hopeful about) was stronger compared to females and other genders. In native English speaking Asians, the connection between getgoing (I just couldn't seem to get going) and lookforward (I felt that I had nothing to look forward to) was stronger compared to all other racial groups. The results from CTree also showed an initial split between native and non-native English speakers, and several further splits with the race variable among native English speakers, but found a split by sexual orientation rather than gender among non-native English speakers.

Both applications suggested significant heterogeneity across multiple different partitioning variables. Without explicitly testing for such heterogeneity, it is possible that erroneous conclusions could have been drawn; using network model trees elucidates areas where heterogeneity is most impactful. Specifically, both applications draw attention to the potential role of English as a native language and gender as important sources of heterogeneity in networks. 


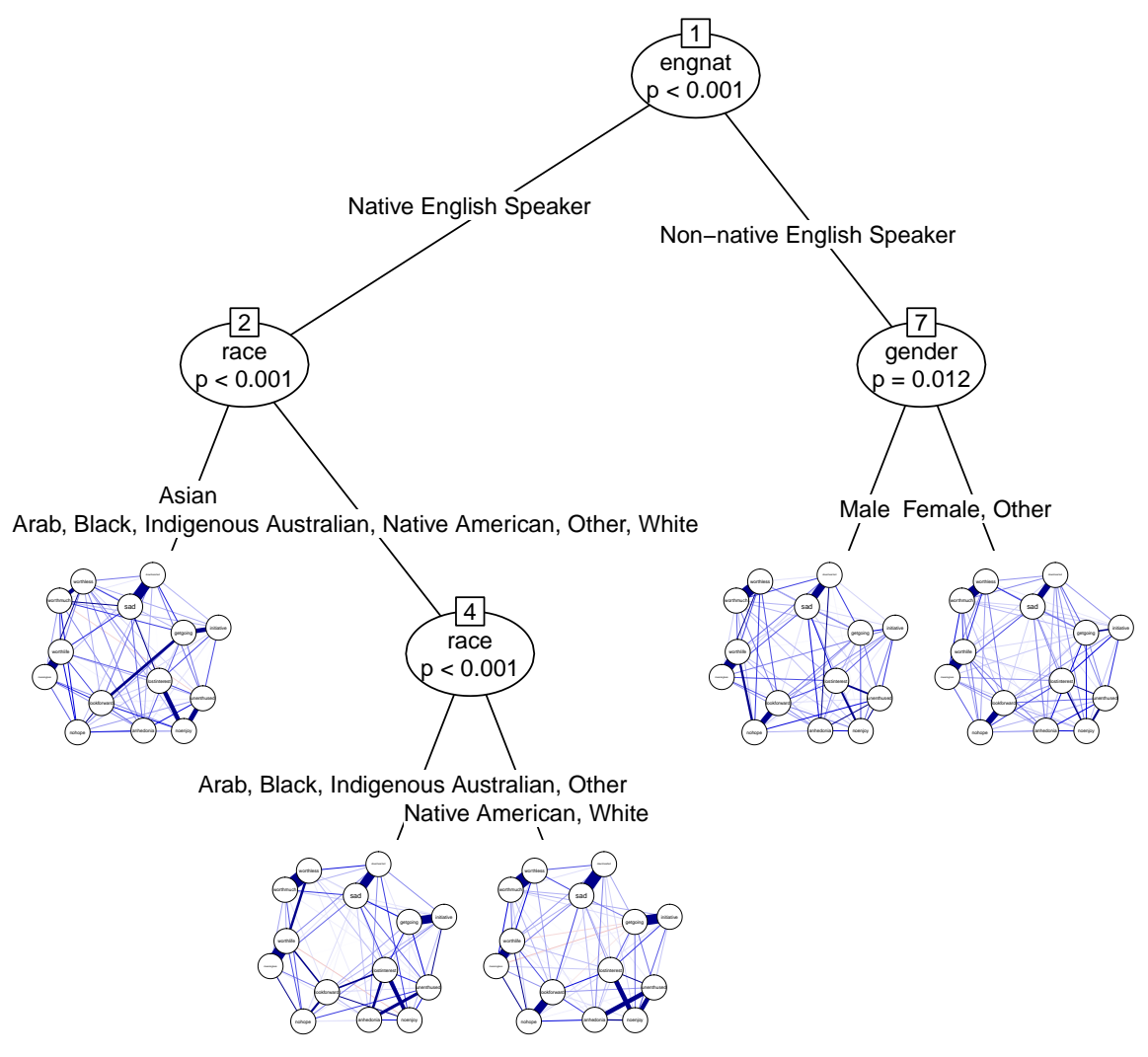

FIGURE 7.

Partitioning DASS networks. Partitioning was done using MOB. Edge thicknesses are determined by the strength of regularized partial correlations between nodes.

\section{Conclusion}

The expanding field of network science shows huge promise in psychology through the application of network psychometrics. One of the challenges in the field has been appropriately modeling heterogeneity in network structure using relevant covariates. We propose a recursive partitioning approach to psychometric networks and introduce two techniques that can accomplish this aim, namely model-based recursive partitioning (MOB) and conditional inference trees (CTree). These methods supplement the toolbox of existing techniques such as moderated network models (MNMs) and changepoint analyses. Simulations show that both $\mathrm{MOB}$ and $\mathrm{CTree}$ demonstrate adequate power to detect medium-to-large effects in datasets representative of those most common in the literature. 
However, there are some limitations to this method. To identify small effects, large sample sizes are necessary. For especially large networks, MOB/CTree may not be viable given the quadratic relationship between the number of nodes and the necessary minimum sample size. Future research could address this problem using a regularization approach such as lasso that reduces the number of estimated parameters. Our simulation approach only evaluated finding a single split in the data; future simulation studies could address situations where multiple hierarchical splits are involved or where other parameters are manipulated such as density values or unequal sample sizes across partitions.

Throughout the paper we treated all variables as metric and applied Pearson correlations. For dichotomous variables, Pearson's $\phi$ coefficient is a popular measure of association between two variables. Since $\phi$ is nothing else than the Pearson correlation formula applied on dichotomous variables, the NMT methodology works in the same way as above. The scores are still meaningful measures, even though the likelihood is misspecified. Having polytomous items, one can think of applying Spearman's correlation. Since the computation of a Spearman correlation amounts to applying Pearson's formula on the rank version of the data, one can transform the original variable scores into ranks and apply NMT as outlined above. Alternatively, other optimal scaling techniques such as correlational aspects (Mair and De Leeuw, 2010) can be used to pre-process the data. In order to use tetrachoric, polychoric, or polyserial correlations, further research is needed since these coefficients are typically found in an iterative manner (see, e.g., Drasgow, 1986).

In summary, recursive partitioning is a powerful addition to the network science toolkit in psychology and has broad applications for addressing heterogeneity in network structures. 


\section{References}

American Psychiatric Association (2013). Diagnostic and Statistical Manual of Mental Disorders (DSM-5囚). American Psychiatric Publishing.

Andrews, D. W. K. (1993). Tests for parameter instability and structural change with unknown change point. Econometrica, 61:821-856.

Borsboom, D. (2017). A network theory of mental disorders. World Psychiatry, 16:5-13.

Brandmaier, A. M., von Oertzen, T., McArdle, J. J., and Lindenberger, U. (2013). Structural equation model trees. Psychological Methods, 18(1):71.

Breiman, L., Friedman, J. H., Olshen, R. A., and Stone, C. J. (1984). Classification and Regression Trees. Chapman \& Hall/CRC, New York.

Brown, T. A., Chorpita, B. F., Korotitsch, W., and Barlow, D. H. (1997). Psychometric properties of the Depression Anxiety Stress Scales (DASS) in clinical samples. Behaviour Research and Therapy, 35:79-89.

Cabrieto, J., Tuerlinckx, F., Kuppens, P., Wilhelm, F. H., Liedlgruber, M., and Ceulemans, E. (2018). Capturing correlation changes by applying kernel change point detection on the running correlations. Information Sciences, 447:117-139.

Costantini, G., Richetin, J., Preti, E., Casini, E., Epskamp, S., and Perugini, M. (2019). Stability and variability of personality networks. a tutorial on recent developments in network psychometrics. Personality and Individual Differences, 136:68-78.

Dalege, J., Borsboom, D., van Harreveld, F., van den Berg, H., Conner, M., and van der Maas, H. L. (2016). Toward a formalized account of attitudes: The Causal Attitude Network (CAN) model. Psychological Review, 123:2-22.

Drasgow, F. (1986). Polychoric and polyserial correlations. In Kotz, S. and Johnson, N. L., editors, Encyclopedia of Statistical Sciences Vol. 7, pages 68-74. Wiley, New York.

Epskamp, S., Borsboom, D., and Fried, E. I. (2018). Estimating psychological networks and their accuracy: A tutorial paper. Behavior Research Methods, 50:195-212.

Epskamp, S., Cramer, A. O. J., Waldorp, L. J., Schmittmann, V. D., and Borsboom, D. (2012). qgraph: Network visualizations of relationships in psychometric data. Journal of Statistical Software, 48(4):1-18.

Epskamp, S., Rhemtulla, M., and Borsboom, D. (2017). Generalized network psychometrics: Combining network and latent variable models. Psychometrika, 82:904-927.

Fokkema, M., Smits, N., Zeileis, A., Hothorn, T., and Kelderman, H. (2018). Detecting treatment-subgroup interactions in clustered data with generalized linear mixed-effects model trees. Behavior Research Methods, 50:2016-2034. 
Fried, E. I. and Nesse, R. M. (2015). Depression is not a consistent syndrome: an investigation of unique symptom patterns in the STAR*D study. Journal of Affective Disorders, 172:96-102.

Friedman, J., Hastie, T., and Tibshirani, R. (2008). Sparse inverse covariance estimation with the graphical lasso. Biostatistics, 9:432-441.

Fritz, J., Fried, E. I., Goodyer, I. M., Wilkinson, P. O., and van Harmelen, A.-L. (2018). A network model of resilience factors for adolescents with and without exposure to childhood adversity. Scientific Reports, 8:15774.

Gosling, S. D., Rentfrow, P. J., and Swann Jr, W. B. (2003). A very brief measure of the Big-Five personality domains. Journal of Research in Personality, 37:504-528.

Hanley, G. P., Iwata, B. A., and McCord, B. E. (2003). Functional analysis of problem behavior: A review. Journal of Applied Behavior Analysis, 36:147-185.

Hansen, B. E. (1997). Approximate asymptotic $p$ values for structural-change tests. Journal of Business \& Economic Statistics, 15:60-67.

Harris, J. K. (2014). An Introduction to Exponential Random Graph Modeling. Sage, Thousand Oaks, CA.

Haslbeck, J., Borsboom, D., and Waldorp, L. (2018). Moderated network models. arXiv preprint arXiv:1807.0287\%.

Haslbeck, J. and Fried, E. I. (2017). How predictable are symptoms in psychopathological networks? A reanalysis of 18 published datasets. Psychological Medicine, 47:2767-2776.

Hjort, N. L. and Koning, A. (2002). Tests for constancy of model parameters over time. Nonparametric Statistics, 14:113-132.

Hothorn, T., Hornik, K., van de Wiel, M. A., and Zeileis, A. (2006a). A lego system for conditional inference. The American Statistician, 60:257-263.

Hothorn, T., Hornik, K., and Zeileis, A. (2006b). Unbiased recursive partitioning: a conditional inference framework. Journal of Computational and Graphical Statistics, 15:651674 .

Hothorn, T. and Zeileis, A. (2015). partykit: A modular toolkit for recursive partytioning in R. Journal of Machine Learning Research, 16:3905-3909.

Jones, P., Simon, T., and Zeileis, A. (2018). networktree: Recursive Partitioning of Network Models. R package version 0.1.1.

Jones, P. J., Heeren, A., and McNally, R. J. (2017). Commentary: A network theory of mental disorders. Frontiers in Psychology, 8:1305. 
Komboz, B., Strobl, C., and Zeileis, A. (2018). Tree-based global model tests for polytomous Rasch models. Educational and Psychological Measurement, 78:128-166.

Mair, P. and De Leeuw, J. (2010). A general framework for multivariate analysis with optimal scaling: The R package aspect. Journal of Statistical Software, 32:1-23.

Marsman, M., Borsboom, D., Kruis, J., Epskamp, S., van Bork, R., Waldorp, L., van der Maas, H., and Maris, G. (2018). An introduction to network psychometrics: Relating ising network models to item response theory models. Multivariate Behavioral Research, $53: 15-35$.

McNally, R. J. (2019). The network takeover reaches psychopathology. Behavioral and Brain Sciences, 42:e15.

Merkle, E. C., Fan, J., and Zeileis, A. (2014). Testing for measurement invariance with respect to an ordinal variable. Psychometrika, 79:569-584.

Merkle, E. C. and Shaffer, V. A. (2011). Binary recursive partitioning methods with application to psychology. British Journal of Mathematical and Statistical Psychology, 64:161181.

Merkle, E. C. and Zeileis, A. (2013). Tests of measurement invariance without subgroups: a generalization of classical methods. Psychometrika, 78:59-82.

Park, J. H. and Sohn, Y. (2019). Detecting structural changes in longitudinal network data. Bayesian Analysis. Forthcoming.

R Development Core Team (2019). R: A Language and Environment for Statistical Computing. R Foundation for Statistical Computing, Vienna, Austria. ISBN 3-900051-07-0.

Salters-Pedneault, K., Tull, M. T., and Roemer, L. (2004). The role of avoidance of emotional material in the anxiety disorders. Applied and Preventive Psychology, 11:95-114.

Schaefer, J., Opgen-Rhein, R., and Strimmer, K. (2015). GeneNet: Modeling and inferring gene networks. $\mathrm{R}$ package version 1.2.13.

Schaefer, J. and Strimmer, K. (2004). An empirical bayes approach to inferring large-scale gene association networks. Bioinformatics, 21:754-764.

Seibold, H., Zeileis, A., and Hothorn, T. (2016). Model-based recursive partitioning for subgroup analyses. The International Journal of Biostatistics, 12:45-63.

Strasser, H. and Weber, C. (1999). On the asymptotic theory of permutation tests. Mathematical Methods of Statistics, 8:220-250.

Strobl, C., Kopf, J., and Zeileis, A. (2015). Rasch trees: A new method for detecting differential item functioning in the Rasch model. Psychometrika, 80:289-316. 
Strobl, C., Malley, J., and Tutz, G. (2009). An introduction to recursive partitioning: rationale, application, and characteristics of classification and regression trees, bagging, and random forests. Psychological Methods, 14:323-348.

Strobl, C., Wickelmaier, F., and Zeileis, A. (2011). Accounting for individual differences in Bradley-Terry models by means of recursive partitioning. Journal of Educational and Behavioral Statistics, 36:135-153.

Wang, T., Merkle, E. C., and Zeileis, A. (2014). Score-based tests of measurement invariance: use in practice. Frontiers in Psychology, 5:1-11.

Wickelmaier, F. and Zeileis, A. (2018). Using recursive partitioning to account for parameter heterogeneity in multinomial processing tree models. Behavior Research Methods, 50:1217-1233.

Zeileis, A. (2006). Implementing a class of structural change tests: an econometric computing approach. Computational Statistics \& Data Analysis, 50:2987-3008.

Zeileis, A. and Hornik, K. (2007). Generalized $M$-fluctuation tests for parameter instability. Statistica Neerlandica, 61:488-508.

Zeileis, A., Hothorn, T., and Hornik, K. (2008). Model-based recursive partitioning. Journal of Computational and Graphical Statistics, 17:492-514. 
Appendix: Score Functions of the Multivariate Gaussian Distribution

We derive the score functions of the multivariate Gaussion distribution for individual observations. In order to fit the networktree model described in the paper, one only requires the score functions of the correlation parameters. However, for the sake of completeness the score functions for all parameters, i.e., mean, standard deviation, and correlations, are derived. The first derivatives of the log-likelihood, as given in Eq. (2), w.r.t. the parameters $\mu_{k}, \sigma_{k}$, and $\rho_{k l}$ are given below. Note that we use the scalar parameter expressions rather than vector notation. The partial derivative w.r.t. $\mu_{k}$ is:

$$
\frac{\partial \ell}{\partial \mu_{k}}=\sum_{l=1}^{p} \varsigma_{k l}\left(y_{l}-\mu_{l}\right)
$$

where $\varsigma_{k l}$ denotes the element in the $k$-th row and $l$-th column of the inverse of the covariance matrix $\boldsymbol{\Sigma}^{-1}$. The partial derivative w.r.t. $\sigma_{k}$ is:

$$
\frac{\partial \ell}{\partial \sigma_{k}}=-\frac{1}{\sigma_{k}}+\frac{1}{\sigma_{k}}\left(\frac{y_{k}-\mu_{k}}{\sigma_{k}}\right) \sum_{l=1}^{p} \omega_{k l}\left(\frac{y_{l}-\mu_{l}}{\sigma_{l}}\right),
$$

where $\omega_{k l}$ denotes the element in the $k$-th row and $l$-th column of the inverse of the correlation matrix $\mathbf{R}^{-1}$. Finally, the partial derivative w.r.t. $\rho_{k l}$ is:

$$
\frac{\partial \ell}{\partial \rho_{k l}}=-\frac{1}{2} \omega_{i j}+\frac{1}{2}\left(\sum_{m=1}^{p} \omega_{k m}\left(\frac{y_{m}-\mu_{m}}{\sigma_{m}}\right)\right)\left(\sum_{m=1}^{p} \omega_{l m}\left(\frac{y_{m}-\mu_{m}}{\sigma_{m}}\right)\right) .
$$

Note that these are the ones used in our NMT approach. 